\title{
A Rare Case of Femoral Neuropathy Associated with Ilio-Psoas Bursitis After 10 Years of Total Hip Arthroplasty
}

\author{
Vivek Singh, Won Yong Shon, Devendra Lakhotia*, Jong Hoon Kim and Tae Wan Kim
}

Department of Orthopaedic Surgery, Korea University College of Medicine, Guro Hospital, 97 Gurodong-gil, Guro-gu, Seoul, 152-703, Korea

\begin{abstract}
We describe a case of femoral nerve palsy caused due to non-infective large iliopsoas bursitis after 10 years of cementless ceramic-on-metal THA. Bursectomy and exploration of femoral nerve were done to relieve the compressive symptoms of femoral nerve. Patient neurological symptoms were recovered within six months. Iliopsoas bursitis after THA can lead to anterior hip pain, lump in inguinal area or abdomen, limb swelling due to venous compression or more rarely neurovascular compressive symptoms depending on size and extension. Treating physician should be aware of this rare condition after THA in the absence of any radiographic findings so that prompt diagnosis and treatment can be carried out.
\end{abstract}

Keywords: Bursectomy, compressive symptoms, exploration, femoral nerve palsy, iliopsoas bursitis, total hip arthroplasty.

\section{INTRODUCTION}

Symptomatic iliopsoas bursitis after total hip arthroplasty (THA) is thought to be caused by foreign-body reaction due to particulate wear debris with or without component loosening or osteolysis [1-5]. In the previous reports describing this condition after THA, presentation was in the form of groin pain or mass or limb swelling secondary to venous occlusion. However, femoral nerve palsy due to illiopsoas bursitis after THA is an infrequently reported condition. We describe a case of right femoral nerve palsy caused due to large iliopsoas bursitis, 10 years following a successful THA.

\section{CASE REPORT}

A fifty-seven-year-old male was treated with THA for primary osteoarthritis of right hip. The bearing surface used in cementless THA was ceramic on metal. He made a good recovery without any complications and remained well for 10 years. In July 2011, he presented to us with the complaint of weakness in his right lower limb for the last one month, and an inability to lift limb for the last two weeks. There was no history of trauma, fever or weight loss. Hip examination revealed no significant findings with a normal range of movements. The patient on neurological assessment, was found to have femoral nerve palsy with weakness of hip flexion and knee extension without any sensory deficit. Distal pulses were normal on both the sides.

Plain radiographs of the hip (Fig. 1) showed no signs of loosening or osteolysis. Lateral inclination and anteversion of acetabular cup were $45^{\circ}$ and $25^{\circ}$ respectively. The

*Address correspondence to this author at the Department of Orthopaedic Surgery, Korea University College of Medicine, Guro Hospital, 97 Gurodong-gil, Guro-gu, Seoul,152-703, Korea; Tel: +82-2-2626-1994;

E-mail: drdevendra.ortho@gmail.com

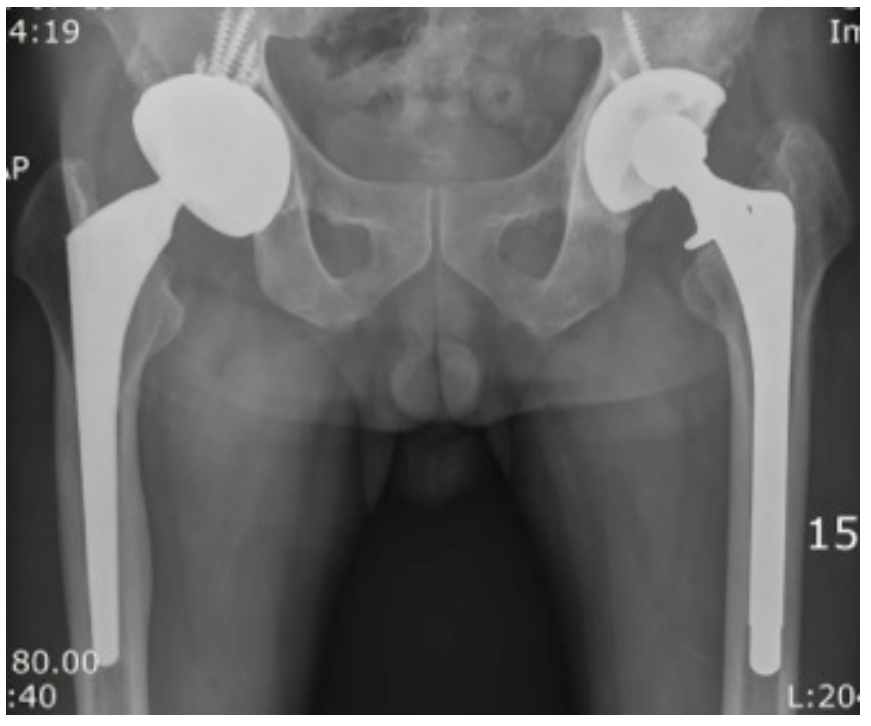

Fig. (1). Plain radiographs of the hip showed no signs of loosening or osteolysis.

femoral stem was in neutral alignment. Blood investigation revealed that complete blood count, ESR and CRP were within normal limits. Serum metal ions were within normal limits. Electromyography and nerve conduction study of right lower limb revealed femoral nerve palsy. Ultrasound examination of the groin demonstrated a large intrapelvic cystic lesion between THA prosthesis and femoral vessels and nerve. Fascicular swelling overlying the femoral nerve was also noted, probably due to mass effect. Fluid-fluid level was also seen within the cystic lesion. There was no definite evidence of intra-articular extension of the lesion to the adjacent hip joint. Ultrasound-guided aspiration was deferred in view of the large size of the lesion, fear of introducing any infective pathogen and risk of recurrence. Further CT evaluation of the right hip confirmed the presence of a large iliopsoas cystic lesion (Fig. 2a, b) along with an overhang of 


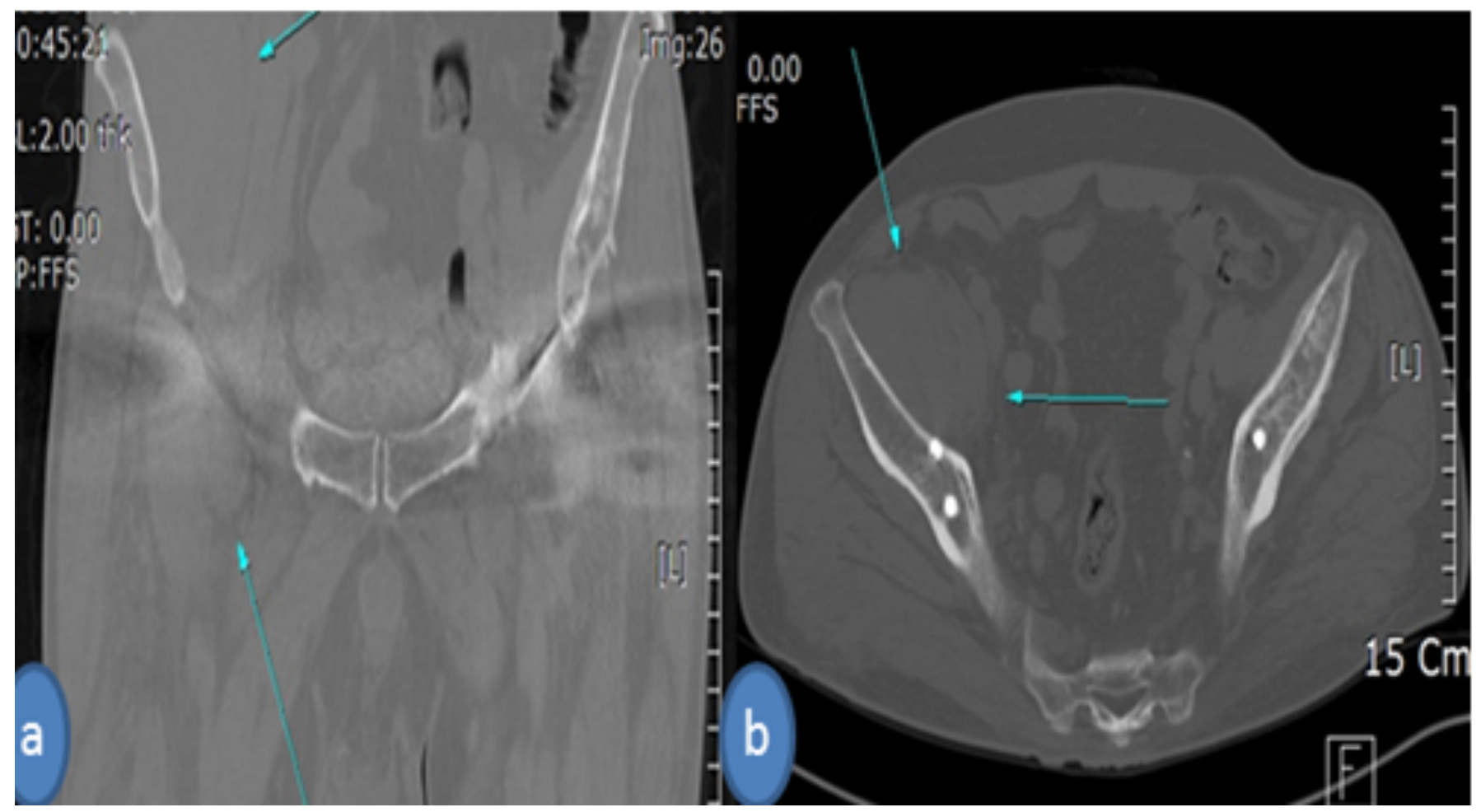

Fig. (2a, b). CT scan of the right hip with a large iliopsoas cystic lesion (indicated by arrows).

anterior portion of the acetabular component beyond the margins of the true acetabulum (Fig. 3). There were no signs of acetabular cup loosening or osteolysis in the CT scan. Lesion size was found to be $7 \mathrm{~cm} \times 4 \mathrm{~cm}$. Based on these findings, we propose that a local reactive response, due to irritation of the iliopsoas bursa caused by the overhanging anterior part of the acetabular component, resulted in the formation of a cyst that secondarily compressed the femoral nerve resulting in neurological signs and symptoms.

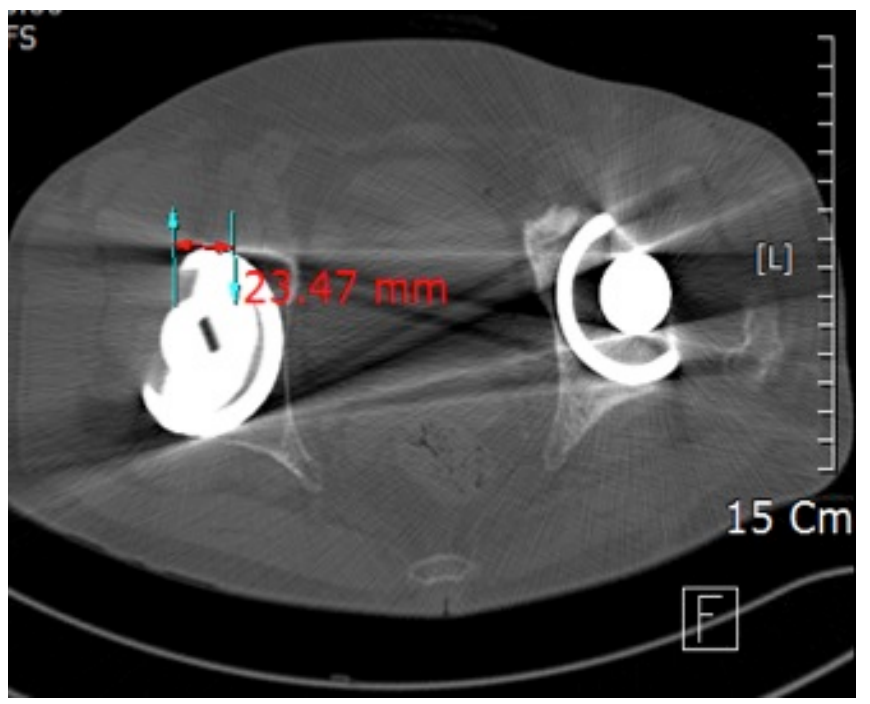

Fig. (3). CT scan showing an overhang of anterior portion of the acetabular component beyond the margins of the true acetabulum.

Exploration of the swelling was carried out using a modified ilio-femoral approach with osteotomy of the anterior superior iliac spine. A dumbbell shaped iliopsoas bursa containing a light brownish inflammatory fluid was identified (Fig. 4). Severe fibrosis and adhesions causing hip impingement were also found around the iliopsoas. On frozen section study, no neoplastic cells or metal debris were identified and neutrophil counts per high power field were not raised. The whole extent of the swelling was identified and excised along with the bursa. Joint capsule was exposed to check for any inflammatory signs. No communication of bursa was found with the hip joint. There was no evidence of wear debris and metallosis in the hip joint. No hip impingement was found after the bursectomy. Exploration and external neurolysis of femoral nerve was performed and the nerve was found in continuity. After thorough irrigation

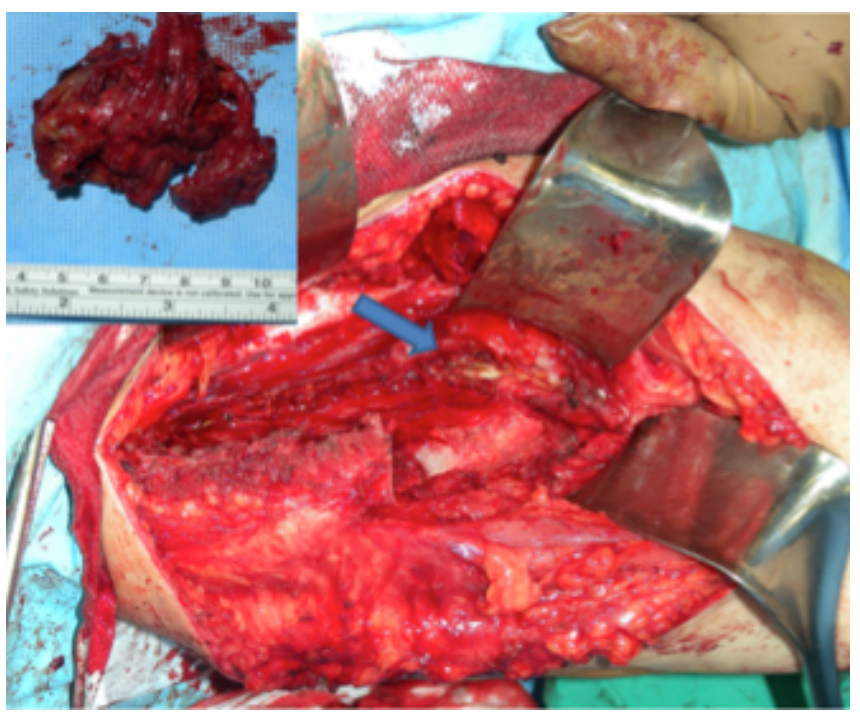

Fig. (4). Modified ilio-femoral approach with bursa (indicated by arrow). Box in the figure indicating tissue after bursectomy. 
and debridement, wound was closed in layers over suction drain. The histopathology revealed chronic inflammation with granulation tissue. There was no foreign body reaction suggesting metal debris. Culture of fluid and tissue was negative.

Postoperatively, a repeat ultrasound done two weeks later and CT scan done eight weeks later showed no cystic collection. The patient was given a hinged knee brace with drop-locks for walking to prevent knee buckling and falls. Review after 6 months showed complete recovery with no neurological symptoms. Another CT scan was done 18 months postoperatively confirming no local recurrence of bursal collection.

Written informed consent was taken from the patient for publication of this case report.

\section{DISCUSSION}

Iliopsoas bursa overlies the anterior aspect of the hip joint capsule, posterior to the iliopsoas tendon \& femoral nerve and lateral to the femoral vessels $[5,6]$. The bursa communicate with the hip joint in $14 \%$ of human population [6] and therefore iliopsoas bursitis is seen in association with conditions that cause chronic inflammation and synovitis of hip joint, such as rheumatoid arthritis and osteoarthritis, and less commonly with synovial chondromatosis, gout, femoral head avascular necrosis and tuberculosis [7-9]. In a series of 14 symptomatic cases of psoas bursitis of all etiologies Meaney et al. found that $40 \%$ of the cases presented with pain, $40 \%$ with a palpable groin mass, and $28 \%$ with lower limb swelling [7]. This condition has also been reported after THA [1-5]. Symptomatic illiopsoas bursitis after THA is a relatively rare condition. The usual cause is foreign-body reaction caused by particulate wear debris in metal-on-metal bearings as well as metal-on-polyethylene articulations [1-5, 10]. Some studies propose that component malposition may lead to edge loading and excessive ion production. Higher wear rates associated with cup inclination greater than $50^{\circ}$ when using metal-on-polyethylene or ceramic-on-ceramic bearings have also been suggested as a cause $[11,12]$.

The usual cause of femoral nerve palsy is intraoperative injury to the nerve which may be caused by retractors placed anterior to the iliopsoas, during anterior capsulectomy or femoral retraction for acetabular preparation. Incidence of overall nerve injury in THA has been reported to be between $1-2 \%$ with sciatic nerve being the most common to be injured and femoral nerve injury being relatively less common [13]. The femoral nerve can also be compressed by extruded cement if acetabular cement pressurization is used. One case of enlarged non-infective iliopsoas bursitis as a cause of femoral nerve palsy one year after THA has been reported [14]. Another case reported illiopsoas bursitis three years after ceramic-on-ceramic THA caused by an inflammatory response to hemorrhage within the bursa [15]. We believe that the bursal swelling in our patient occurred as a reactive phenomenon in response to the overhanging anterior portion of the acetabular component which might have irritated the overlying ilio-psoas tendon. Ultrasonography is recommended as the first-line investigation, since it is simple, quick and cost-effective [7, $8,9,16]$. CT and MRI may further define local anatomy within the pelvis aiding in diagnosis and preoperative planning $[1,7,8,16]$.

Although uncommon, it should be recognized that iliopsoas bursitis may occur as a complication of THA and should be considered in the differential diagnosis when presented with compressive neurovascular symptoms. Treatment may involve aspiration or excision. We believe that surgical exploration should be considered if there are significant compressive symptoms. In our case, due to the large size of the cystic swelling and significant symptoms, we decided to excise it by a modified ilio-femoral approach. If the cause is thought to be wear debris then some authors advocate the need for complete excision through a separate approach, usually an ilioinguinal approach combined with revision surgery that can be staged or performed simultaneously $[1,4,5,17]$. There were no radiologic features of loosening, infection or component malposition in this case so we did not attempt to revise the THA. The exact cause of illiopsoas bursitis after 10 years of THA could not be determined. After ruling out other causes of illiopsoas bursitis like foreign body reaction to wear debris, the most likely explanation is the irritation of the iliopsoas bursa caused by an overhanging anterior part of the acetabular component. We recommend that care should be taken during the primary procedure so as to select the appropriate size of the acetabular component which does not irritate any intrapelvic structures.

\section{CONCLUSION}

A large iliopsoas bursitis after THA can lead to severe neurovascular compressive symptoms in the absence of any radiographic findings. Treating physician should be aware of this condition so that prompt diagnosis and treatment can be carried out.

\section{ABBREVIATION}

THA $=\quad$ Total hip arthroplasty

\section{CONFLICT OF INTEREST}

The authors confirm that this article content has no conflict of interest.

\section{ACKNOWLEDGEMENTS}

Declared none.

\section{REFERENCES}

[1] Yang SS, Bronson MJ. Cystic enlargement of the iliopsoas bursa causing venous obstruction as a complication of total hip arthroplasty. A case report. J Arthroplasty 1993; 8(6): 657-61.

[2] Goyal S, Moss MC, Breusch SJ. Venous outflow obstruction 8 years following Ring total hip arthroplasty. A rare complication of acetabular loosening and polyethylene wear. Acta Orthop Scand 1997; 68(1): 67-9.

[3] Cheung YM, Gupte CM, Beverly MJ. Iliopsoas bursitis following total hip replacement. Arch Orthop Trauma Surg 2004; 124(10): 720-23.

[4] Beksaç B, Tözün R, Baktiroglu S, Sener N, Gonzalez Della Valle A. Extravascular compression of the femoral vein due to wear debris-induced iliopsoas bursitis: a rare cause of leg swelling after total hip arthroplasty. J Arthroplasty 2007; 22(3): 453-6. 
[5] Matsumoto K, Hukuda S, Nishioka J, Fujita T. Iliopsoas bursal distension caused by acetabular loosening after total hip arthroplasty. A rare complication of total hip arthroplasty. Clin Orthop Relat Res 1992; 279: 144-8.

[6] Chandler SB. The iliopsoas bursa in man. Anat Rec 1934; 58(3): 235-40.

[7] Meaney JF, Cassar-Pullicino VN, Etherington R, Ritchies DA, McCal IW, Whitehouse GH. Ilio-psoas bursa enlargement. Clin Radiol 1992; 45(3): 161-8.

[8] Ginesty E, Dromer C, Galy-Fourcade D, et al. Iliopsoas bursopathies. A review of twelve cases. Rev Rhum Eng Ed 1998; 65(3): 181-6

[9] Bianchi S, Martinoli C, Keller A, Bianchi-Zamorani MP. Giant iliopsoas bursitis: sonographic findings with magneticresonance correlations. J Clin Ultrasound 2002; 30(7): 437-41.

[10] Maurer-Ertl W, Friesenbichler J, Liegl-Atzwanger B, Kuerzl G, Windhager R, Leithner A. Noninflammatory pseudotumor simulating venous thrombosis after metal-on-metal hip resurfacing. Orthopedics 2011; 34(10): 678-81.

[11] Schmalzried TP, Guttmann D, Grecula M, Amstutz HC. The relationship between the design, position, and articular wear of ac- etabular components inserted without cement and the development of pelvic osteolysis. J Bone Joint Surg Am 1994; 76(5): 677-88.

[12] Schmalzried TP, Shepherd EF, Dorey FJ, et al. The John Charnley Award. Wear is a function of use, not time. Clin Orthop Relat Res 2000; (381): 36-46.

[13] DeHart MM, Riley LH Jr. Nerve injuries in total hip arthroplasty. J Am Acad Orthop Surg 1999; 7(2): 101-11.

[14] Liman J, von Gottberg P, Bahr M, Kermer P. Femoral nerve palsy caused by ileopectineal bursitis after total hip replacement: a case report. J Med Case Rep 2011; 5: 190.

[15] Park KS, Diwanji SR, Kim HK, Song EK, Yoon TR. Hemorrhagic iliopsoas bursitis complicating well-functioning ceramic-onceramic total hip arthroplasty. J Arthroplasty 2009; 24(5): 826.e1e5.

[16] Wunderbaldinger P, Bremer C, Schellenberger E, Cejna M, Turetschek K, Kainberger F. Imaging features of iliopsoasbursitis. Eur Radiol 2002; 12(2): 409-15.

[17] Madan S, Jowett RL, Goodwin MI. Recurrent intrapelvic cyst complicating metal-on-metal cemented total hip arthroplasty. Arch Orthop Trauma Surg 2000; 120(9): 508-10. 